\title{
Canadian integrated program for antimicrobial resistance surveillance: Retail food highlights, 2003-2012
}

\author{
Avery $B P^{1^{*}}$, Parmley $E J^{1}$, Reid-Smith $R J^{1}$, Daignault $D^{2}$, Finley $R L^{3}$, Irwin $R J^{1}$ \\ 1 Laboratory for Foodborne Zoonoses, Public Health Agency of Canada, Guelph, ON \\ 2 Laboratory for Foodborne Zoonoses, Public Health Agency of Canada, Saint Hyacinthe, QC \\ ${ }^{3}$ Centre for Food-borne, Environmental and Zoonotic Infectious Diseases, Public Health Agency of Canada, Guelph, \\ ON \\ *Corresponding author: brent.avery@phac-aspc.gc.ca
}

\begin{abstract}
Background: The Canadian Integrated Program for Antimicrobial Resistance Surveillance (CIPARS) is a collaborative, integrated program designed to track antimicrobial resistance (AMR) among enteric bacteria isolated from various livestock commodities along the food-producing continuum ("farm to fork") and in humans.

Objective: To provide a summary of the prevalence and trends in AMR among select bacteria isolated from raw, fresh chicken, pork, and beef in 2012 at the retail food level and to link these data with other findings from CIPARS.
\end{abstract}

Methods: Meat samples were collected from randomly selected geographic areas across Canada weighted by population for subsequent isolation of bacteria and interpretation of the associated AMR profiles. Salmonella, Campylobacter and generic Escherichia coli (E. coli) were tested in chicken, and $E$. coli was tested in beef and pork. Data were analyzed for 2012 and temporal and regional trends were examined between 2003 and 2012 by province/region.

Results: Overall, resistance levels to Salmonella in retail chicken varied widely by region and year. For example, ceftiofur resistance to Salmonella in retail chicken was significantly lower in 2012 than in 2004 in Ontario and in Québec; however, among all regions sampled, resistance was significantly higher in 2012 compared to 2006. Across all regions sampled, resistance to Campylobacter in retail chicken was relatively low in $2012(<16 \%)$ with the exception of tetracycline resistance. In 2012, ciprofloxacin resistance to Campylobacter in chicken declined in British Columbia but significantly increased in Ontario, compared to 2011. In 2012, $\beta$-lactam resistance to $E$. coli in retail beef remained low ( $\leq 1 \%)$ and was also relatively low comparable to previous years in pork.

Conclusion: In Canada, as is the case worldwide, there is evidence of resistance to medically important antimicrobials among bacteria from retail meats. Resistance among organisms isolated from poultry, beef, and pork at the retail food level is characterized by wide variation over time and across different regions.

\section{Introduction}

The Canadian Integrated Program for Antimicrobial Resistance Surveillance (CIPARS) is a collaborative, integrated program designed to track antimicrobial resistance (AMR) among enteric bacteria isolated from various livestock commodities along the food-producing continuum ("farm to fork") and in humans. Specifically, the majority of the surveillance activities are strategically conducted at the farm, abattoir, and retail food levels, as well as in clinical isolates from animals and humans. Antimicrobials are frequently used in veterinary medicine and livestock production; some of the same antimicrobials, or close relatives, are also used in human medicine. All antimicrobial use in humans and animals can select for resistant 
strains of bacteria; concerns are typically focused around uses that are, or are perceived to be, inappropriate as these unnecessarily add to the burden of resistance. The use of these important drugs in food animals (e.g., chickens, pigs, cattle) may pose a risk to public health through potential transfer of antimicrobial resistant organisms or resistance genes in food.

The Retail Meat Surveillance component of CIPARS provides data on antimicrobial resistance among select bacteria found in raw meat at the provincial/regional level. Retail food represents a logical sampling point for surveillance of AMR because it is the endpoint of food animal production, and thus is indicative of human exposure. Retail surveillance provides a measure of human exposure to resistant bacteria through consumption of fresh, raw (uncooked) meat products from selected commodities.

Annual CIPARS reports are published on a regular basis. The objective of these reports is to provide a summary of the prevalence and trends (temporal variations) in antimicrobial resistance in select bacterial species isolated from humans and the agri-food sector. In the 2012 surveillance year, the CIPARS Annual Report was refined to include multiple chapters released in succession to make the CIPARS data available to stakeholders in a more timely fashion (1). This article summarizes some of the notable retail meat findings of the most recent 2012 CIPARS Report and links these data with findings from other CIPARS surveillance components.

\section{Methods}

The commodities of interest for retail meat surveillance reported here were raw meat products most commonly consumed by Canadians. These commodities and the products sampled by CIPARS included poultry (chicken legs or wings [skin-on]), pork (chops), and beef (ground). Turkey (ground) was also added in 2012 but with only one year of surveillance, data were not included in this summary. The unit of analysis was the bacterial isolate recovered from raw meat. Bacteria of interest in chicken were the pathogens Campylobacter and Salmonella, and generic Escherichia coli (E. coli), as an indicator of AMR selection pressure and a reservoir of resistance genes. From beef and pork, given the low prevalence of Campylobacter and Salmonella in these commodities at retail as determined in earlier years of the Program, only $E$. coli was routinely cultured and then tested for antimicrobial susceptibility. Retail meat samples are submitted from randomly selected geographic areas (i.e., census divisions defined by Statistics Canada), weighted by population, in each participating province/region. In 2012, retail meat samples were collected on a weekly basis in Ontario and Québec, and every other week in British Columbia, Saskatchewan, and the Maritime provinces (New Brunswick, Nova Scotia, and Prince Edward Island).

Prevalence estimates, based on an expected yield of 100 isolates of each targeted bacteria per commodity per province/region per year, are used to determine the number of samples to be collected. As sampling was less frequent in British Columbia, Saskatchewan, and the Maritimes, the target of 100 isolates per year was not always achieved in those provinces/regions in a given year.

Resistance is reported based on Health Canada's Categorization of antimicrobial drugs (2): Category I (very high importance to human medicine), Category II (high importance to human medicine), and Category III (moderate importance to human medicine). Retail data were analyzed and results compared and integrated with the other components of CIPARS (e.g., farm, abattoir, and human) to provide a more complete picture of antimicrobial resistance in food-borne bacteria in Canada. Full details about CIPARS including sampling, laboratory and analytical methods are available in the Annual Reports (3). 


\section{Results}

\section{Chicken}

Across all provinces sampled, the top three chicken Salmonella serovars were $S$. Heidelberg, $S$. Kentucky, and $S$. Enteritidis. All $S$. Enteritidis isolates were susceptible to all antimicrobials tested in 2012. No ciprofloxacin (Category I) resistance was observed in any serotype in 2012. Nalidixic acid (Category II) resistance was observed in two S. Kentucky isolates (4\%) from British Columbia; previously nalidixic acid resistance had only been observed in two isolates from Saskatchewan in 2005 (Figure 1). Overall, Category I $\beta$-lactam (amoxicillin-clavulanic acid, ceftriaxone, ceftiofur) resistance levels (26\%) remained similar to levels in 2011 (30\%). Resistance to ceftiofur was significantly lower (23\%) in 2012 than $2004(46 \%)$ in Ontario. Although resistance to ceftiofur in Québec was significantly lower $(28 \%)$ in 2012 than 2003 (50\%), resistance was significantly higher in 2012 compared to 2006 (9\%) (Figure 1).

FIGURE 1. Temporal variation in resistance to selected antimicrobials in Salmonella isolates from chicken, CIPARS Retail Meat Surveillance, 2003-2012

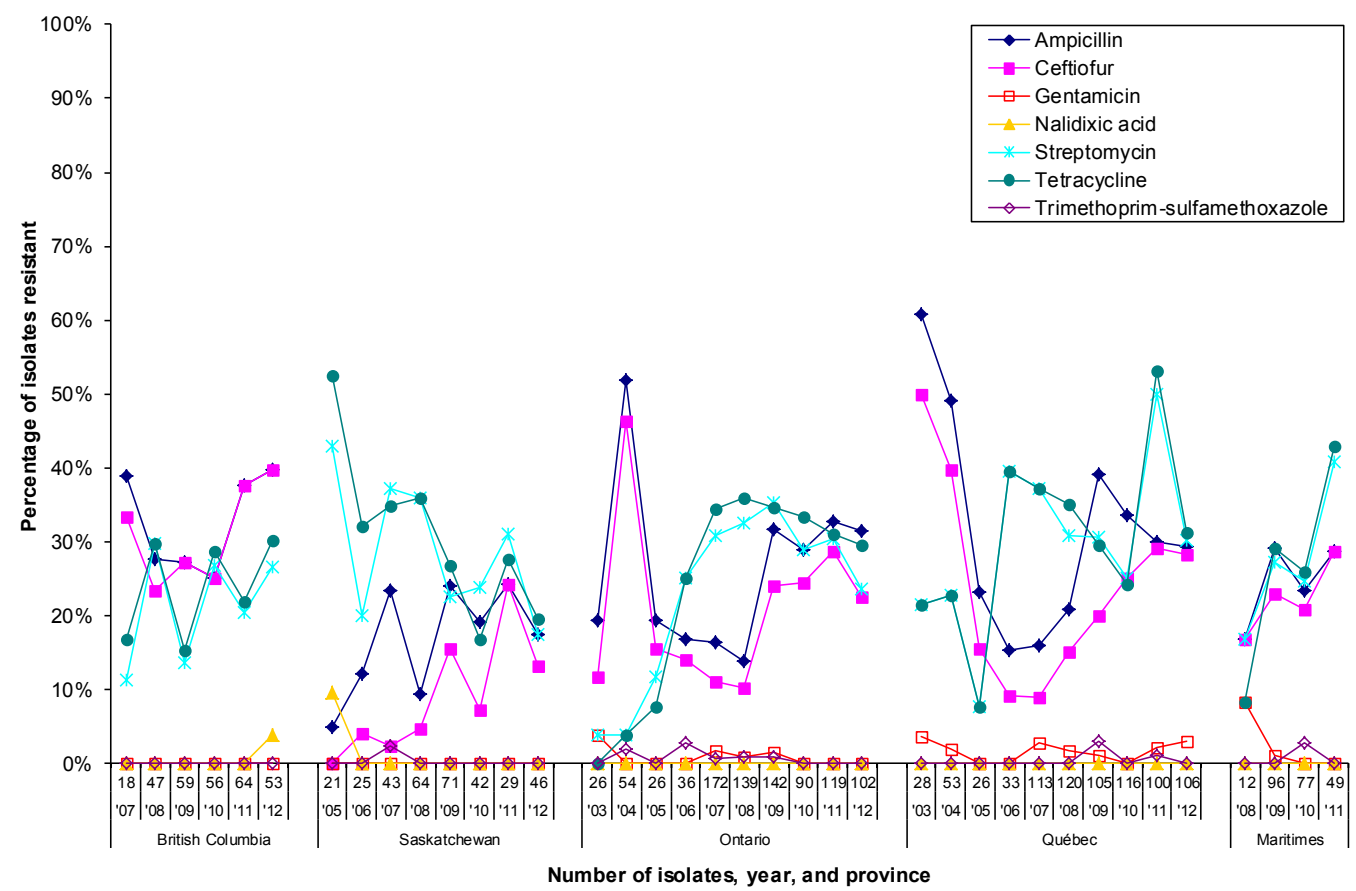

The Maritimes area is sampled as a region (New Brunswick, Nova Scotia, Prince Edward Island). Due to unforeseen and protracted lengthy delays in retail sampling in the Maritimes in 2012, in the interest of precision, the data are incomplete and not presented for this year. Data for this region will be presented again in 2013.

Resistance among Campylobacter from retail chicken was relatively low in 2012 (<16\%) with the exception of tetracycline resistance. Ciprofloxacin resistance continued to decline in British Columbia in $2012(8 \%)$ compared to $2011(13 \%)$, and remained at a similar level in Saskatchewan (5\%) compared to 2011 (4\%). In Ontario, ciprofloxacin resistance significantly increased in 2012 (16\%) compared to 2003 (4\%). More details are available in the case study by Agunos et al., in this issue (4). Resistance to azithromycin (Category II) was significantly lower in 2012 (8\%) than in 2003 (22\%) in Québec (Figure 2). 
FIGURE 2. Temporal variation in resistance to selected antimicrobials in Campylobacter isolates from chicken, CIPARS Retail Meat Surveillance, 2003-2012

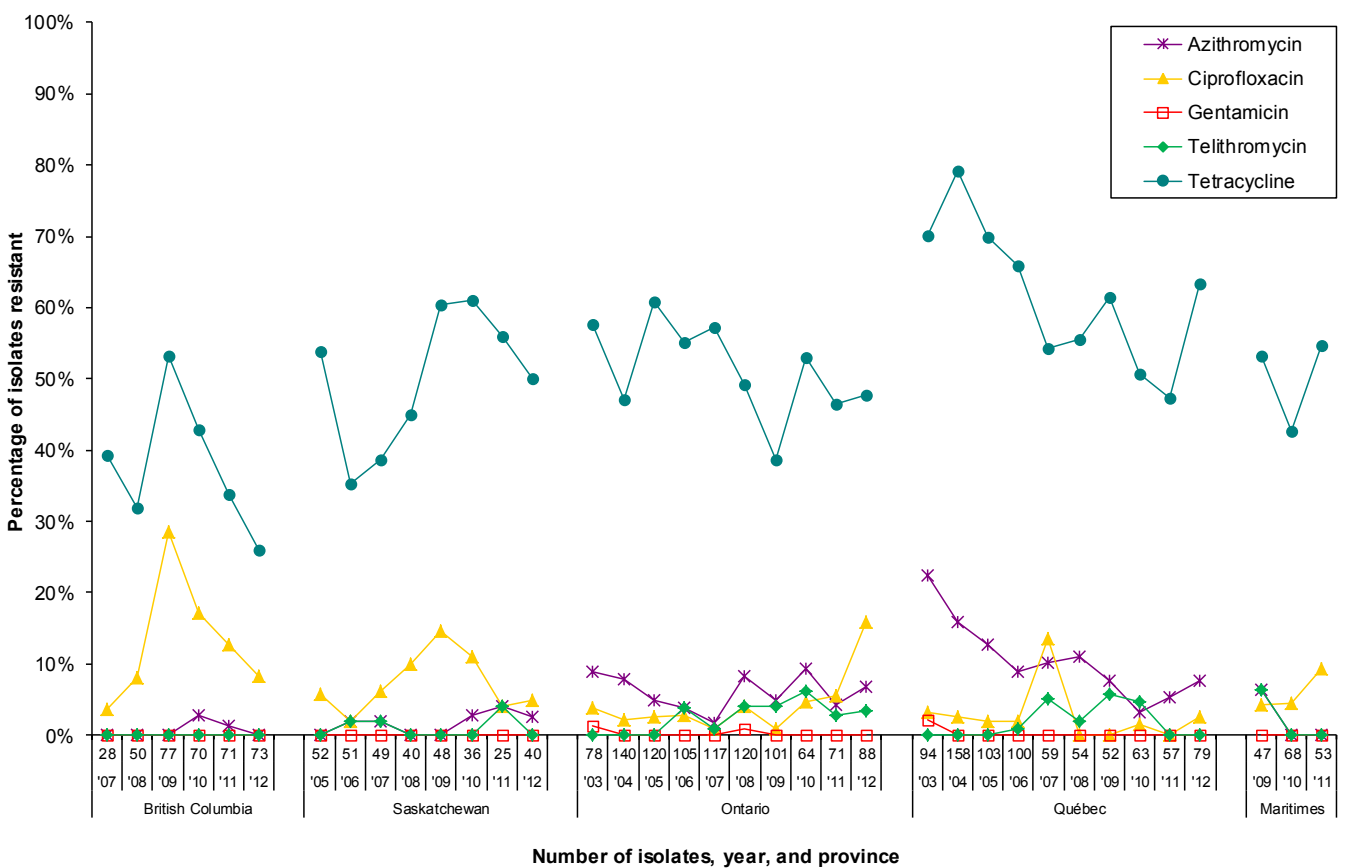

The Maritimes area is sampled as a region (New Brunswick, Nova Scotia, Prince Edward Island). Although routine retail surveillance began in the Maritime region in 2008, no results are displayed for that year due to concerns regarding harmonization of laboratory methods.

Due to unforeseen and protracted lengthy delays in retail sampling in the Maritimes in 2012, in the interest of precision, the data are incomplete and not presented for this year. Data for this region will be presented again in 2013.

No ciprofloxacin resistance was observed in retail chicken E. coli isolates in 2012. Overall, resistance to Category I $\beta$-lactams remained similar to those in 2011. In Saskatchewan, resistance to ceftiofur was significantly higher in $2012(22 \%)$ than in $2005(4 \%)$, and in Québec, resistance to ceftiofur was also significantly higher in $2012(25 \%)$ than in $2006(6 \%)$.

\section{Pork and beef}

As in previous years, resistance to Category I $\beta$-lactams remained low $(\leq 1 \%)$ in beef $E$. coli isolates in 2012. No ciprofloxacin resistance was observed in 2012. Similarly, among generic $E$. coli isolates from pork, resistance levels of the Category I $\beta$-lactams, including ceftiofur, remained at comparable levels to previous years in each region.

\section{Discussion}

In Canada, as is the case worldwide (5), there is evidence of resistance to medically important antimicrobials among bacteria from retail meats. Resistance among organisms isolated from poultry, beef and pork at retail is characterized by wide variation over time and across different regions. A key strength of the CIPARS system is that data from its various components (e.g., farm, clinical animal, abattoir, retail, human) can be analyzed, compared and integrated together to better understand the epidemiology of 
antimicrobial resistance in food-borne bacteria in Canada. Two notable and relatively recently identified integrated issues are described below.

\section{Ceftiofur resistance in Salmonella Heidelberg}

Ceftiofur is used to treat and prevent a wide variety of infectious animal diseases. Although it is not labelled for use in chickens in Canada, it has been used to control E. coli omphlitis (yolk sack infection) in broiler chicks. Due to the mechanism of resistance, if a bacterium is resistant to ceftiofur, it is almost always resistant to ceftriaxone and all other third generation cephalosporins. Ceftriaxone is one of the drugs of choice for treating severe salmonellosis and other food-borne bacterial infections in pregnant women and children.

Changes in the level of ceftiofur resistance in S. Heidelberg were observed between 2003 and 2008 (6). More recent data from 2011 and 2012 show that, regardless of the source of S. Heidelberg (human or retail meat), resistance to Category I $\beta$-lactams (specifically ceftiofur and ceftriaxone) remained relatively high (Figure 3). Across Canada, $27 \%$ of human S. Heidelberg isolates were resistant to both ceftiofur and ceftriaxone in 2012. Although $S$. Heidelberg was less frequently recovered from people in Western Canada, the proportion of isolates resistant to ceftiofur and ceftriaxone was higher: $51 \%$ of $S$. Heidelberg isolates from people in the four western provinces demonstrated resistance, whereas $21 \%$ of isolates from the eastern provinces of Canada were resistant.

As in previous years, resistance to ceftiofur and ceftriaxone was also commonly observed in $S$. Heidelberg isolates from agri-food sources in 2012. Among S. Heidelberg isolates from retail chicken meat, $32 \%(30 / 94)$ were resistant to ceftiofur and ceftriaxone; by region, resistance ranged from $0 \%$ in Saskatchewan to $86 \%$ in British Columbia (Figure 3). As in people, S. Heidelberg was more commonly observed in chicken meat from Eastern Canada. Although much less common in Western Canada, a higher percentage of the isolates demonstrate resistance to Category I $\beta$-lactams.

FIGURE 3. Temporal (2003-2012) variation in ceftiofur resistance in Salmonella Heidelberg isolates from retail chicken and humans

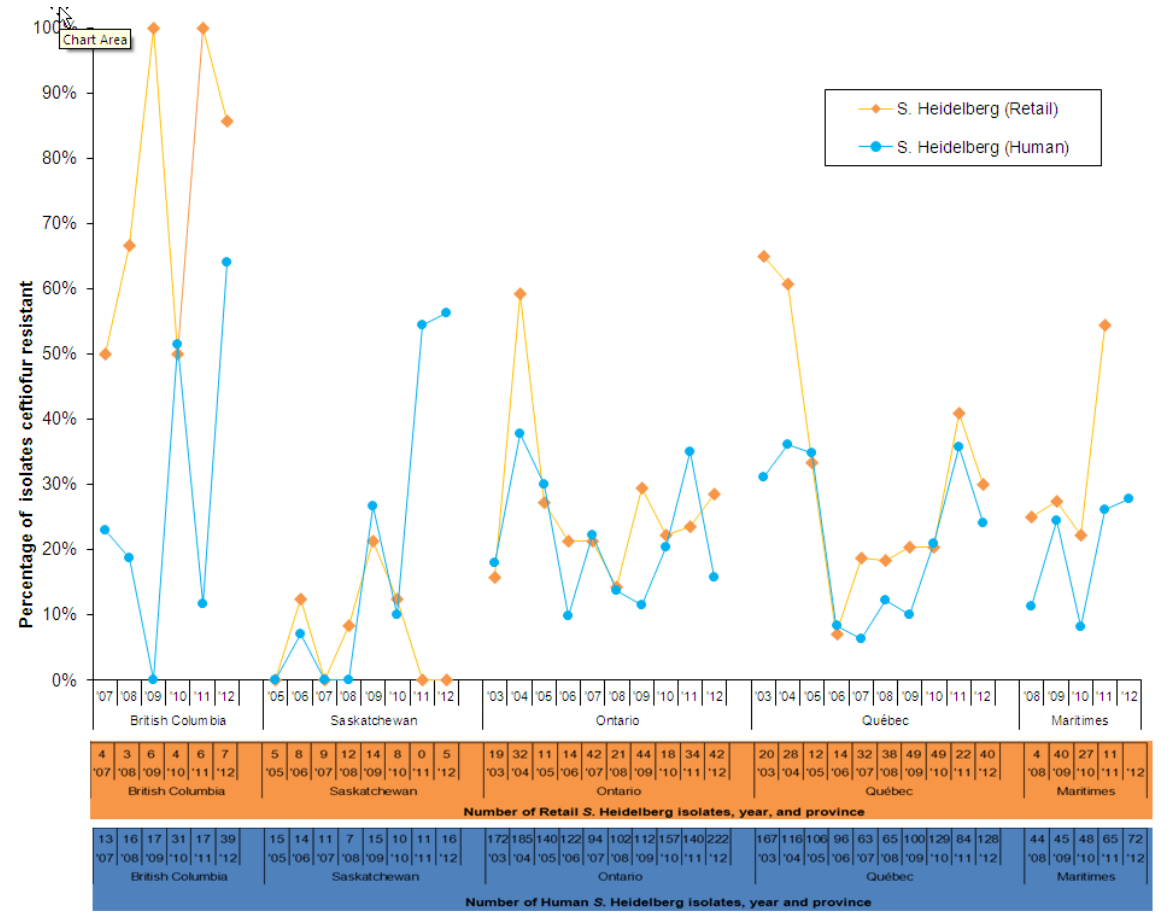




\section{Ciprofloxacin resistance in Campylobacter}

Over the past several years, ciprofloxacin resistance has been observed in Campylobacter isolates, particularly those from retail chicken $(7,8)$. As noted earlier, important and changing regional differences in the level of resistance were observed in 2012 (Figure 4). In British Columbia, the proportion of ciprofloxacin-resistant Campylobacter continued to decline in 2012 (8\%), down from a high of 29\% in 2009. In 2012, Ontario had the highest proportion (16\%) of ciprofloxacin-resistant Campylobacter from retail chicken in that year; this is the highest level of ciprofloxacin resistance observed to date in the province. More information about this important health issue and how it is influencing surveillance and policy decisions in Canada is presented by Agunos et al., in this issue (4).

Ciprofloxacin resistance in Campylobacter was also observed in isolates recovered from samples collected at slaughter in 2012. Ciprofloxacin resistance was observed in $6 \%(8 / 152)$ of isolates from cattle, $7 \%(11 / 155)$ of chicken isolates, and $10 \%(28 / 287)$ of pig isolates. It is important to note that abattoir isolates are recovered from samples of cecal contents and meant to be more reflective of the bacterial population on the farm rather than contamination at the slaughter plant. Slightly higher level of resistance in isolates from pigs may be attributed to the species of Campylobacter recovered (C. coli is the most common species in pigs; $C$. jejuni, is the most common species in chicken).

FIGURE 4. Temporal variation in resistance to ciprofloxacin in Campylobacter isolates from chicken; CIPARS Retail Meat Surveillance, 2003-2012

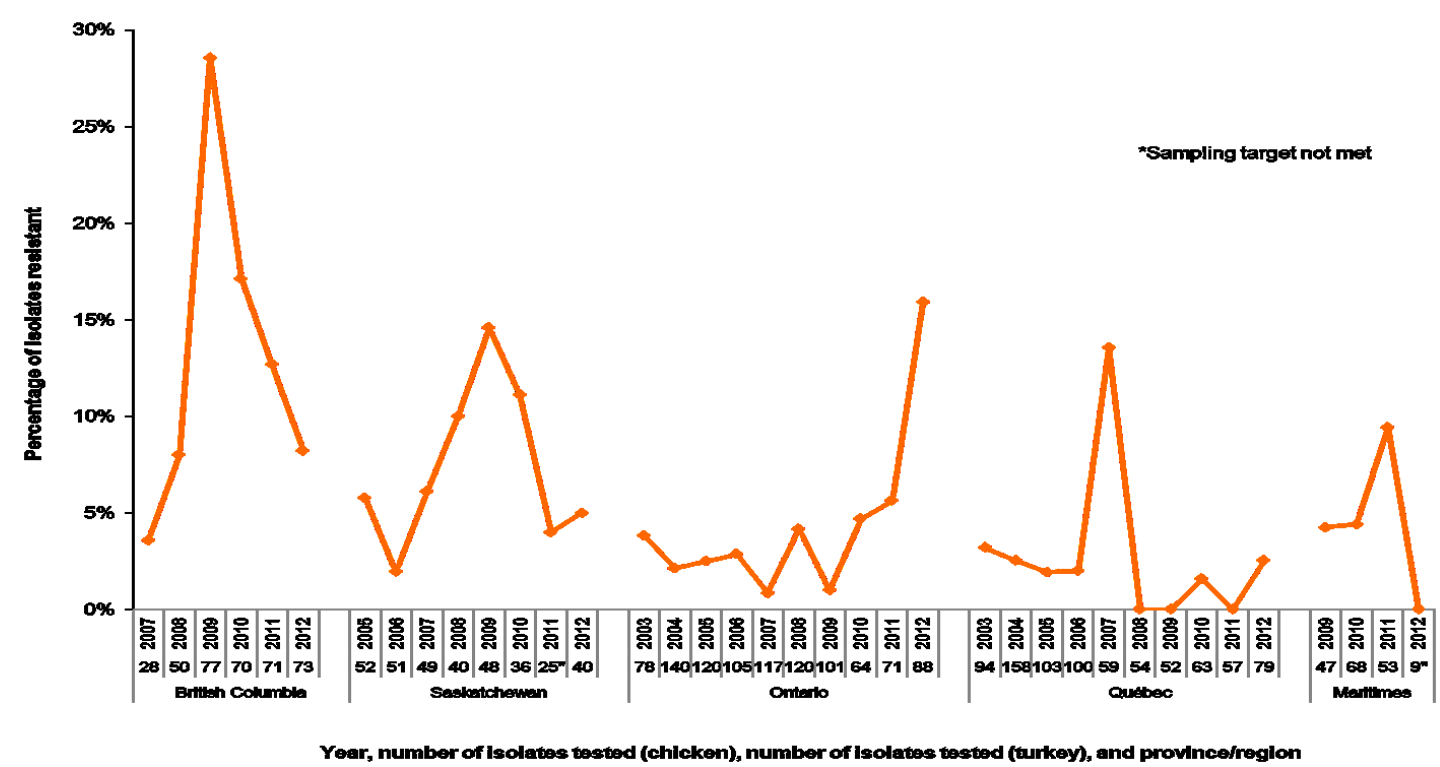

\section{Conclusion}

Antimicrobial resistance will continue to challenge the health of Canadians and of people around the world for some time to come. CIPARS data is used to detect changes in resistance over time and across Canada, and to help inform the development of evidence-based policies on antimicrobial use in hospital, community, and agricultural settings. Ongoing collection of surveillance data will help document the effectiveness of these changes to prolong the effectiveness of antimicrobial drugs. 


\section{Acknowledgements}

The authors would like to acknowledge the effort of the CIPARS retail field workers, sampling node coordinators, and laboratory technicians for their contributions. We would also like to thank the participating health units and environmental health officers/public health inspectors for sampling in remote areas in British Columbia and Ontario, and the provincial public health laboratories for their support of the human component of CIPARS. Lastly, the authors would like to acknowledge the University of Prince Edward Island for retail sampling and laboratory support in the Maritime provinces. The careful collection of samples, processing of isolates, and recording of results are essential to the ongoing success of CIPARS.

\section{Conflict of interest}

None.

\section{References}

(1) Public Health Agency of Canada (PHAC). Canadian Integrated Program for Antimicrobial Resistance Surveillance (CIPARS) 2012 Annual Report-Chapter 2. Antimicrobial Resistance. Guelph, ON: PHAC; 2014. http://www.phac-aspc.gc.ca/cipars-picra/2012/annu-report-rapport-eng.php

(2) Government of Canada [web page]. Health Canada, Veterinary Drugs Directorate. Categorization of antimicrobial drugs based on importance in human medicine. 2009 Apr. http://www.hc-sc.gc.ca/dhpmps/vet/antimicrob/amr_ram_hum-med-rev-eng.php

(3) Government of Canada [web page]. Public Health Agency of Canada. Canadian Integrated Program for Antimicrobial Resistance Surveillance (CIPARS) Reports. 2014 Sep. http://www.phac-aspc.gc.ca/ciparspicra/pubs-eng.php

(4) Agunos A, Léger D, Avery BP, Parmley J, Deckert A, Carson CA, Reid-Smith RJ, Irwin, RJ. Ciprofloxacinresistant Campylobacter in broiler chicken in Canada. CCDR. 2014 Nov 6; 40 S-2:38-42.

(5) WHO Advisory Group on Integrated Surveillance of Antimicrobial Resistance. Tackling foodborne antimicrobial resistance through integrated surveillance-Report of the 3rd meeting of the WHO Advisory Group on Integrated Surveillance of Antimicrobial Resistance, 14-17 June 2011, Oslo, Norway. Geneva: World Health Organization; 2012. http://apps.who.int/iris/bitstream/10665/75198/1/9789241504010_eng.pdf?ua=1

(6) Dutil L, Irwin R, Finle R, Ng LK, Avery B, Boerlin P, Bourgault A-M, Cole L, Daignault D, Desruisseau A, Demczuk W, Hoang L, Horsman G, Ismail J, Jamieson F, Maki A, Pacagnella A, Pillai D. Ceftiofur resistance in Salmonella enterica serovar Heidelberg from chicken meat and humans, Canada. Emerging Infectious Diseases. 2010; 16:48-54.

(7) Government of Canada [web page]. Public Health Agency of Canada, Canadian Integrated Program for Antimicrobial Resistance Surveillance (CIPARS). Surveillance Bulletin: Emergence of ciprofloxacin-resistant Campylobacter in retail chicken in British Columbia and Saskatchewan. 2011 Nov.: http://www.phacaspc.gc.ca/cipars-picra/bulletin-eng.php

(8) Agunos A, Léger D, Avery BP, Parmley EJ, Deckert A, Carson CA, Dutil L. Ciprofloxacin-resistant Campylobacter spp. in retail chicken, western Canada. Emerging Infectious Diseases. 2013; 19(7):1121-4. 